\title{
Kinetic Simulations of Type II Radio Burst Emission Processes
}

\author{
Urs Ganse $^{1}$, Felix Spanier ${ }^{1}$ and Rami Vainio ${ }^{2}$ \\ ${ }^{1}$ Lehrstuhl für Astronomie, Universität Würzburg, \\ Am Hubland, 97074 Würzburg, Germany \\ email: uganse@astro.uni-wuerzburg.de \\ email: fspanier@astro.uni-wuerzburg.de \\ ${ }^{2}$ Department of Astronomy, University of Helsinki \\ email: rami.vainio@helsinki.fi
}

\begin{abstract}
Using a fully relativistic, 3D particle in cell code we have studied Langmuir- and electromagnetic wave processes in a CME foreshock plasma with counterstreaming electron beams. Langmuir wave excitation in resonance with the plasma frequency is observed, with timescales in accordance with theoretical predictions. However, no three wave interaction leading to emission of electromagnetic waves were detectable within the timeframe of our simulations.
\end{abstract}

Keywords. methods: numerical, radiation mechanisms: nonthermal, shock waves, Sun: radio radiation, waves

\section{Introduction}

Since the beginning of solar radio observations in the 1950ies, transient radio phenomena, so called solar radio bursts, have been observed. While consistent theories for the emission mechanism of most radio burst types have been found in the past, the so called type II radio bursts (correlated with coronal mass ejections) emission processes are still insufficiently explained.

Since CMEs are very extended structures whose shock fronts span areas from the low corona out towards interplanetary space, the large range of plasma densities should lead to a correspondingly large variation in plasma frequency, and hence, broadband emission. Observations however show a narrowband structure of two emission peaks, one approximately at the plasma frequency near the tip of the CME shock front (the so called fundamental emission), and one at its first harmonic.

\section{The Model}

In a model suggested by Cairns et al. (2003) emission is limited to a small spatial area in the foreshock of the CME, where electrons accelerated by shock drift acceleration at a quasi-perpendicular point along the curved shock front form an electron beam in the otherwise quiescent, thermal foreshock solar wind.

By a beam-driven instability, the electrons excite Langmuir waves, which can then undergo three-wave interaction processes similar to the creation of type III bursts (Melrose, 1986).

In addition to analytical calculation (Li et al. 2004), there are multiple in-situ spacecraft measurements of foreshock regions for interplanetary shocks (Pulupa and Bale, 2008) which confirm the existence of electron beams and Langmuir wave creation.

Using fundamental plasma simulations, we are trying to confirm this picture of the type II radio burst emission process. Similar simulations with reduced complexity have been 


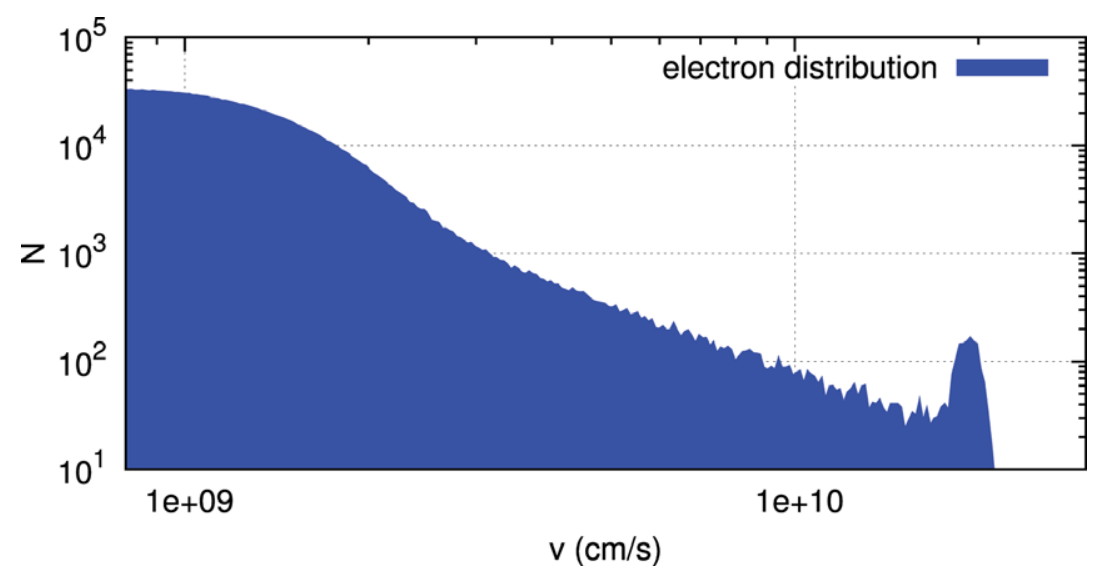

Figure 1. Model velocity distribution of electrons in the CME foreshock region: in addition to the thermal bulk, a superthermal electron beam component at 5 thermal velocities is present.

performed in 1D (Karlický and Vandas, 2007), but no complete 3-dimensional simulations exist to this date.

\section{PiC simulations using ACRONYM}

The numerical simulations for this research were conducted using ACRONYM, the particle in cell code developed at the university of Würzburg during the last 3 years. It is a fully-relativistic, second order $3 \mathrm{D} \mathrm{PiC}$-code written in $\mathrm{C}++$, with MPI parallelization and excellent scaling behaviour even on large supercomputers.

Since the PiC method requires the plasma Debye length to be resolved, simulations encompassing the complete CME are unfeasible. Instead, the simulation scenario is focussed on the microphysics within the foreshock region. A homogeneous solar wind plasma fills the box, with two counterstreaming electron beams added to create an approximation of the electron distribution function (see fig. 1).

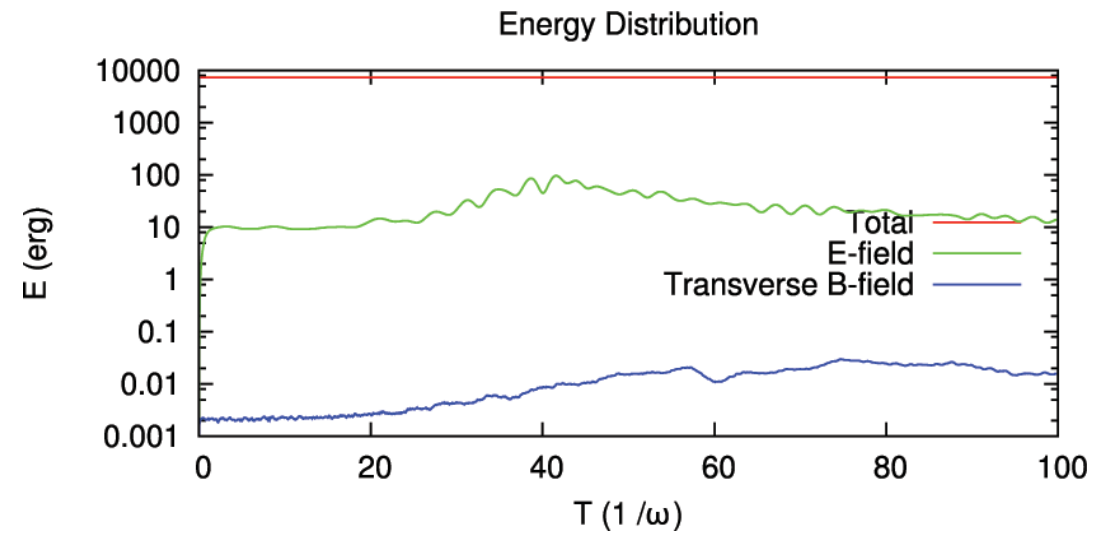

Figure 2. Evolution of energy distribution in the simulation. Kinetic energy of the electron beams is converted into electric fields, when Langmuir waves are excited. The decay of these waves deposits energy into the transverse B-field ( $B_{x}$ and $B_{y}$ component).

Input parameters for the simulation were a plasma frequency of $200 \mathrm{MHz}$, a temperature of $10^{6} \mathrm{~K}$, background magnetic field of $|\mathbf{B}|=0.1 \mathrm{G}$ parallel to the electron beam direction, the correct physical mass ratio of $m_{p} / m_{e}=1836$ and density of $2.5 \cdot 10^{7} \mathrm{~cm}^{-3}$. 
Simulation sizes varied from $256 \times 256 \times 512$ cells (elongated in streaming direction) to $768^{3}$ cells, with 20 particles per cell. With a Debye length of $\lambda_{D}=1.35 \mathrm{~cm}$, this leads to physical extents of up to $10 \times 10 \times 10 \mathrm{~m}$.

\section{Results and Conclusion}
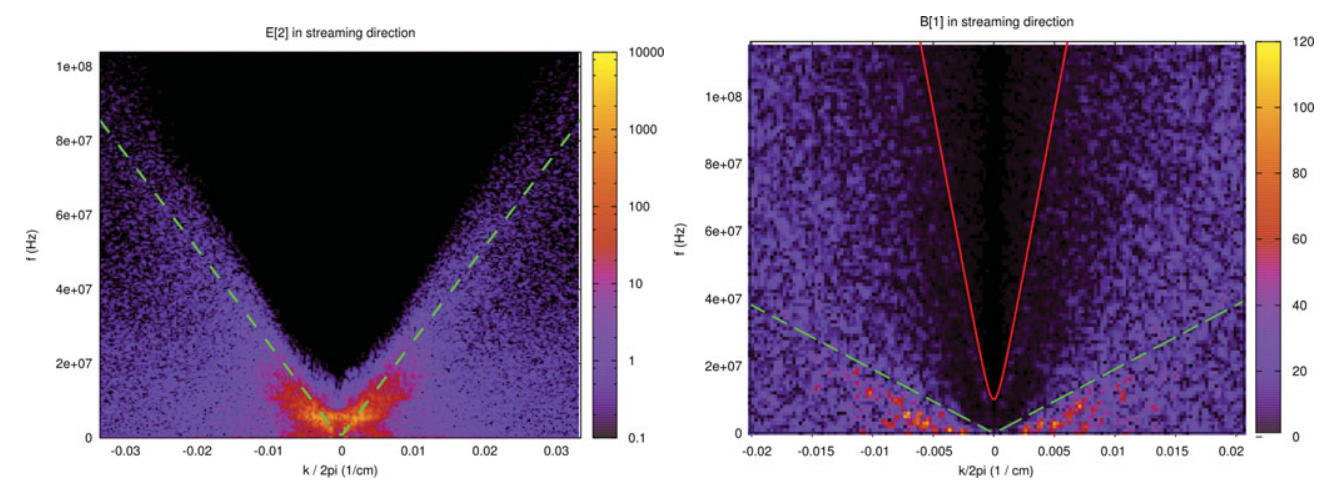

Figure 3. Dispersion plots (created using temporal and spatial fourier transform) of the longitudinal component of the electric field (left) and a transverse component of the magnetic field (right) along the streaming direction. A strong signal of Langmuir waves is visible near resonance with the plasma frequency. No electromagnetic emissions of large intensity are detectable. The green line represents the beam velocity of the electrons, the red line indicates the theoretical dispersion relation of the electromagnetic mode.

In the energy distribution (see fig. 2), a conversion of particle kinetic energy into electric field energy is observed. The dispersion plot of the longitudinal electric field along the streaming direction (see the left side of fig. 3) confirms a strong signal of Langmuir waves near resonance with the plasma frequency.

Creation of electromagnetic waves within the simulation timeframe is not visible. Instead, rise in magnetic energy (in the components perpendicular to the background field and streaming velocity) appears in figure 2. Both dispersion and magnetization of this wave indicate that this is a beam driven mode (Wiles \& Cairns, 2000).

The quick decay of the Langmuir wave excitation, visible in the energy plot, suggests that other interaction processes than the one leading to radio emission may be dominant in our simulations, hence making radio emissions invisible. A possible reason is the high numerical noise in the PiC-code, leading to overly strong density fluctuations. Work is now ongoing to reduce these fluctuations (by increasing particle number and improved smoothing) and verify their effect on Langmuir wave stability.

\section{Acknowledgements}

We thank the Jülich supercomputing centre for the grant of computing time on Jugene. UG acknowledges support by the Elite Network of Bavaria. FS acknowledges support by the German Science Foundation, Grant SP1124/3.

\section{References}

Cairns, I., Knock, Robinson, P., \& Kuncic Space Sci. Revs., Vol. 107, April (2003), p. 27-34.

M. Karlický \& M. Vandas Planet. and Space Sci., Vol. 55, December (2007), p. 2336-2339.

Li, B., Willes, A., Robinson, P., \& Cairns, I. APS Meeting Abstracts, Nov (2004), p. 1035P-+. Melrose, D. B. Instabilities in Space and Laboratory Plasmas, Cambridge Univ. Press, Aug (1986) Pulupa \& Bale 2008, ApJ, Vol. 676, April (2008), p. 1330-1337.

Willes, A. \& Cairns, I. Physics of Plasmas, Vol. 7, Aug (2000), p. 3167-3180. 